\title{
Accelerate the Face Detection Optimization with Edge Detection and the Discrete Cosine Transform (DCT)
}

\author{
Javad Haddadnia, Ph.D \\ Hakim Sabzevari University \\ Iran,Sabzevar
}

\author{
Mojtaba Farzaneh \\ Hakim Sabzevari University \\ Iran,Sabzevar
}

\author{
Armin Parsian nejad \\ Hakim Sabzevari University \\ Iran,Sabzevar
}

\begin{abstract}
This study presents a new method for accelerating and optimizing face detection, while preserving a high level of accuracy. This method uses local features that are extracted using block-base discrete cosine transform (DCT). This study uses edge detection method for face recognition with ICA algorithms. The technique used for edge detection is Laplacian of Gaussian (LOG). To find objects, face position and local information the discrete cosine transform is used. Here the main idea is edge detection and finding face position in the picture for DCT processing. Edge detection was applied for accelerating image processing. In this paper we use CohnKanade AU-Coded Facial Expression Database.
\end{abstract}

\section{Keywords}

Face Detection, DCT, Edge detection, image processing, LOG.

\section{INTRODUCTION}

Image representation is one of the key issues in the computer vision and image processing communities. Face recognition has been an active research area over the last 30 years. Scientists from different areas of psychophysical sciences and those from different areas of computer sciences have worked on it. Psychologists and neuroscientists mainly deal with the human perception part of the topic, whereas engineers studying on machine recognition of human faces deal with the computational aspects of face recognition. Face recognition is mainly applied in the fields of biometrics, access control, law enforcement, and security and surveillance systems. Biometrics deals with methods to automatically verify or identify individuals, using their physiological or behavioral characteristics [1-6]. At first a LOG algorithm is used to detect the Edges, and then the edges of the image are used to determine the exact location of the face, when the specified location is calculated in the original picture, the coordinates are selected. Finally the image processing method (DCT) is applied for further analysis. [7]

\section{LAPLACIAN EDGE DETECTION}

Given an image matrix, the Laplacian of the image function is the second order partial derivatives along $\mathrm{x}$ and $\mathrm{y}$ directions. [8].

$\nabla^{2} g=\frac{\partial^{2} g}{\partial x^{2}}+\frac{\partial^{2} g}{\partial y^{2}}(1)$

In the digital approximation of the second order, partial derivative in $\mathrm{x}$ direction is

$$
\begin{aligned}
& \frac{\partial^{2} g}{\partial x^{2}}=\frac{\partial\{g(r, c)-g(r, c-1)\}}{\partial x}(2) \\
& =\frac{\partial g(r, c)}{\partial x}-\frac{\partial g(r, c-1)}{\partial x}(3)
\end{aligned}
$$

$$
\begin{aligned}
& =g(r, c)-g(r, c-1)-g(r, c-1)+g(r, c-2)(4) \\
& =g(r, c)-2 g(r, c-1)+g(r, c-2)(5)
\end{aligned}
$$

Similarly, the second-order derivative along y direction is given by,

$\frac{\partial^{2} g}{\partial y^{2}}=g(r, c)-2 g(r-1, c)+g(r-2, c)(6)$

both the approximating Eqs. (5) and (6) are centered around the point $(\mathrm{r}-1, \mathrm{c}-1)$. Conveniently, this pixel could be replaced by $(\mathrm{r}, \mathrm{c})$. [9-11]

\subsection{Laplacian of Gaussian Edge Detector}

The weight distribution in a Laplacian mask evokes strong response to stray noise pixels. It indicates that some sort of noise cleaning preceded by Laplacian should provide a better result. For noise cleaning, one may employ Gaussian smoothing. Then the resultant algorithm is LOG edge operator. [9-13] The algorithm steps are given as follows:

1. Smooth the image intensity $\mathrm{g}(\mathrm{r}, \mathrm{c})$ by convolving it with a digital mask corresponding to Gaussian function.

2. Apply the Laplacian mask on the smooth image intensity profile.

3. Find the zero crossing in the image subjected to Laplacian second derivative operator.

Mathematically,

$g^{\prime \prime}(r, c)=\nabla^{2}\{g(r, c) \times G(r, c)\} \quad(7)$

which, following the rule of convolution, becomes

$g^{\prime \prime}(r, c)=g(r, c) \times\left\{\nabla^{2} G(r, c)\right\}(8)$

Extracting edge points or edges by detecting zero crossing in the second derivative still suffers from the problem of false alarm i.e., a non-edge pixel may be marked as edge pixel. This is because even a small nonlinear variation in intensity profile gives rise to zero crossing in second-order derivative. The problem may be surmounted by considering both firstand second-order derivatives. In that case, an edges is said to be present if there exists a zero crossing in second. [9-13]

The input facial images and edge-detected face images using LOG is presented in Figure 1.

\section{DISCRETE COSINE TRANSFORM}

The discrete cosine transform (DCT) is a useful tool in signal processing for frequency analysis. Its results correspond to the frequency components in an (signal) image - i.e., the low and high frequencies are arranged in DCT components. The DCT represents a sequence of finite pixel elements in terms of the sum of cosine functions oscillating at different frequencies. [14-16] For a 2D image of size $\mathrm{M}^{*} \mathrm{~N}$, the DCT-II is defined 
as:

$C(a, b)=\alpha(a) \alpha(b) \sum_{x=0}^{M-1} \sum_{y=0}^{N-1} f(x, y) C_{x}^{M}(a) C_{y}^{N}(b)(9)$

with its inverse transform:

$f(x, y)=\sum_{a=0}^{M-1} \sum_{b=0}^{N-1} \alpha(a) \alpha(b) C(a, b) C_{x}^{M}(a) C_{y}^{N}(b)(10)$

Where $C_{k}^{P}(h)=\cos \left[\frac{\pi(2 k+1) h}{2 P}\right]$ For both equations (9) and (10), $\quad \alpha(a)$ and $\alpha(b)$ are defined as $\alpha(a)=1 / \sqrt{M} \quad$ if $\mathrm{a}=0$, otherwise $\alpha(a)=\sqrt{2 / M}$ and $\alpha(b)=1 / \sqrt{N}$ if $\mathrm{b}=0$ otherwise $\alpha(b)=\sqrt{2 / N}$ if $\mathrm{a}=0$ and $\mathrm{b}=0 \quad$ then $\quad \mathrm{C}(0,0)$ is $(1 / \sqrt{M})(1 / \sqrt{N}) \sum_{x=0}^{M-1} \sum_{y=0}^{N-1} f(x, y)$ which is an average value of the sample sequences. Therefore, the suppression is on the DC and a first few AC components and is defined as a divisor matrix $\mathrm{Q}$ with: [14-16]

$$
\begin{gathered}
Q(0,0)=\beta_{0} \\
Q(0,1)=Q(1,0)=\beta_{1} \\
Q(0,2)=Q(2,0)=Q(2,2)=\beta_{2}
\end{gathered}
$$

And

$$
Q(0,3)=Q(3,0)=Q(2,3)=Q(3,2)=\beta_{3}
$$

with all other $\mathrm{Q}(\mathrm{a}, \mathrm{b}) \mathrm{s}$ set to 1 , where

$\beta_{0} \in[0.85,1.3], \beta_{1} \in[0.5,0.7], \beta_{2}=\beta_{1}+0.1$

and $\beta_{3}=\beta_{2}+0.1$ We define the quantized DCT face image as:

$D(x, y)=i D C T\left(\frac{D C T(f(x, y))}{Q(x, y)}\right)(11)$

where DCT and iDCT are the discrete cosine transform and its inverse as defined in equations (9) and (10). Using DCT in conjunction with TV is useful insofar as the facial features are enhanced while the illumination variations are suppressed. [6],[17-20].

\section{FIGURES/CAPTIONS}

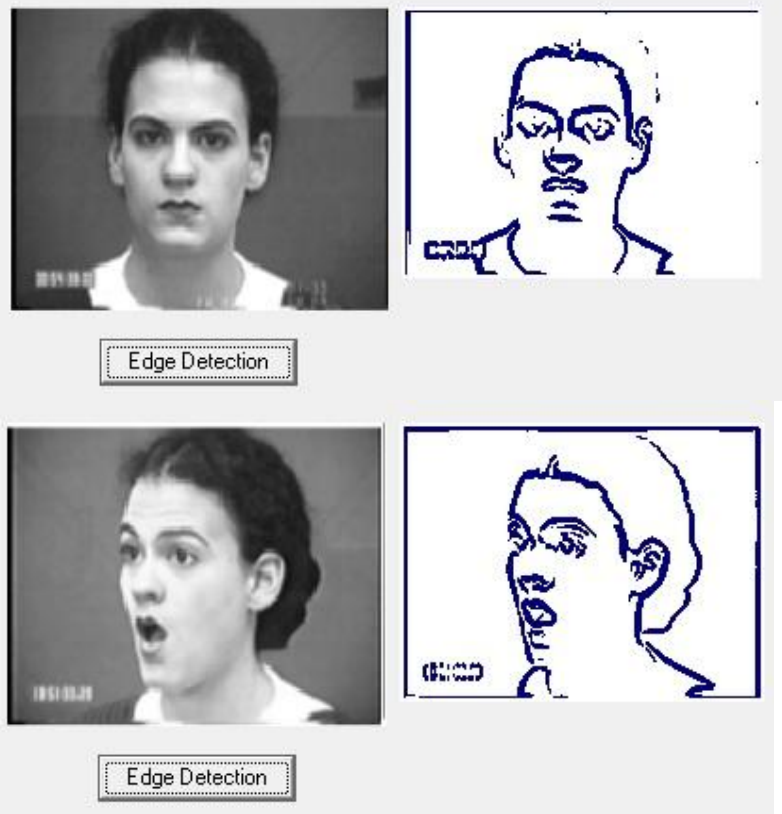

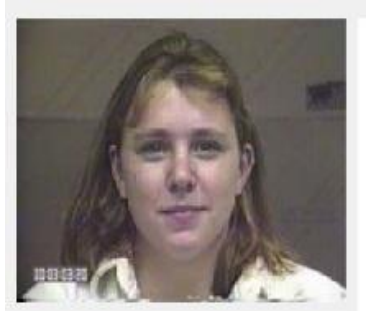
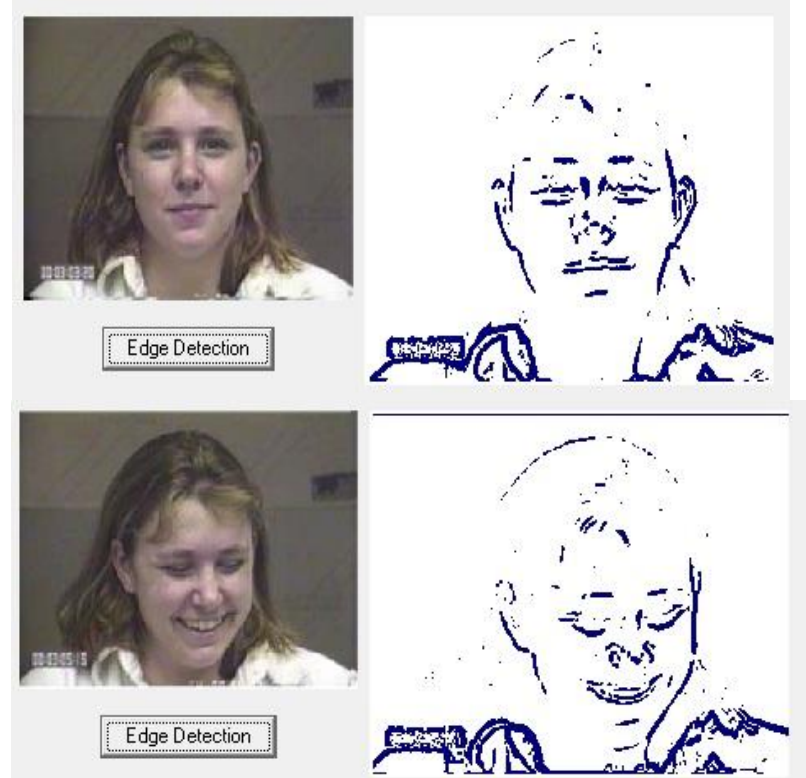

Fig 1: Edge Detection

Figure1 is Input face images and Edge detected images by LOG operator.
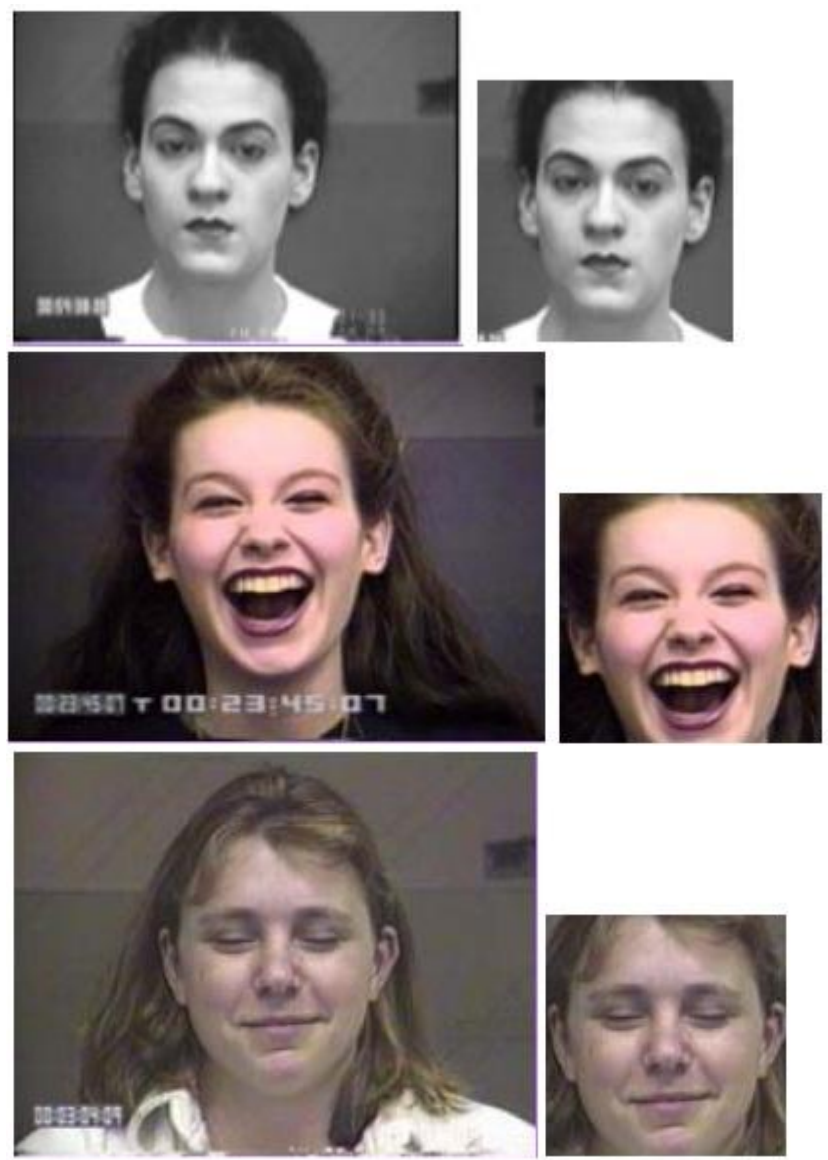

Fig 2: Our algorithm (use Edge Detection and DCT)

\section{RESULT}

Whit Analysis of sections 2 and 3 of the image Face detection and DCT, we recognize the Control and reduce processing time. The results of this study are in Table 1 
Table 1.Recognition performance (\%)

\begin{tabular}{|c|c|c|c|}
\hline Graphics & Accuracy & Delay & $\begin{array}{c}\text { Process } \\
\text { Result }\end{array}$ \\
\hline DCT & 83.3 & More than our & True \\
\hline OUR & 100 & Fast & True \\
\hline
\end{tabular}

\section{CONCLUSION}

As discussed above, through the use of the proposed method in which the focus is on edge detection and face localization, determining the coordinates of face and selecting it from the original image, the processing time and size of the mathematical calculations are noticeably reduced. Regarding the sections two and three in this paper, the mathematical algorithm for edge detection is more compact and faster in this method in comparison with the DCT method. This method reduces the image coordinates and only selects the face from the image. As a result processing images needs much less time and is fulfilled much faster. This algorithm Accelerate the Face Detection Optimization with Edge detection and The Discrete Cosine Transform (DCT).

\section{REFERENCES}

[1] R. Gottumukkal, V.K. Asari, -An Improved Face Recognition Technique Based On Modular Pca Approachll,Pattern Recognition Letters, 25(4), 2004.

[2] B. Heisele Et Al, -Face Recognition: Component-Based Versus Global Approachesll, Computer Vision And Image Understanding, 91:6-21, 2003

[3] T. Kim Et Al, -Component-Based Lda Face Description For Image Retrieval And Mpeg-7 Standardisationl, Image And Vision Computing, 23(7):631-642, 2005.

[4] A.Pentland, B. Moghaddam, T. Starner And M. Turk,-View Based And Modular Eigenspaces For Face Recognitionll, Proceedings Of Ieee Cvpr, Pp. 84-91, 1994.

[5] Vijay, B., and A. Nagabhushana Rao. "Analysis Of Face Recognition-A Case Study On Feature Selection And Feature Normalization." Analysis 3.3 (2013): 971-979.

[6] Brunelli, Roberto, and Tomaso Poggio. "Face recognition: Features versus templates." Pattern Analysis and Machine Intelligence, IEEE Transactions on 15.10 (1993): 1042-1052.

[7] Ahmed, Nasir, T. Natarajan, and Kamisetty R. Rao. "Discrete cosine transform." Computers, IEEE Transactions on 100.1 (1974): 90-93.
[8] Van Dokkum, Pieter G. "Cosmic-Ray Rejection by Laplacian Edge Detection." Publications of the Astronomical Society of the Pacific 113.789 (2001): $1420-1427$.

[9] Karande, Kailash Jagannath, and Sanjay Nilkanth Talbar. "Laplacian of Gaussian Edge Detection for Face Recognition Using ICA." Independent Component Analysis of Edge Information for Face Recognition. Springer India, 2014. 35-47.

[10] Dollár, Piotr, et al. "Behavior recognition via sparse spatio-temporal features." Visual Surveillance and Performance Evaluation of Tracking and Surveillance, 2005. 2nd Joint IEEE International Workshop on. IEEE, 2005.

[11] Yang, Ming-Hsuan, David J. Kriegman, and Narendra Ahuja. "Detecting faces in images: A survey." Pattern Analysis and Machine Intelligence, IEEE Transactions on 24.1 (2002): 34-58.

[12] Jain, Ramesh, Rangachar Kasturi, and Brian G. Schunck. Machine vision. Vol. 5. New York: McGraw-Hill, 1995.

[13] Canny, John. "A computational approach to edge detection." Pattern Analysis and Machine Intelligence, IEEE Transactions on 6 (1986): 679-698.

[14] Pernebo, Lars. "An algebraic theory for design of controllers for linear multivariable systems--Part I: Structure matrices and feedforward design." Automatic Control, IEEE Transactions on 26.1 (1981): 171-182.

[15] Celler, Frank, et al. "Generating random elements of a finite group." Communications in algebra 23.13 (1995): 4931-4948.

[16] Lukáš, Jan, and Jessica Fridrich. "Estimation of primary quantization matrix in double compressed JPEG images." Proc. Digital Forensic Research Workshop. 2003.

[17] Srisuk, Sanun. "Robust Face Recognition Based On Texture Analysis." Int J Adv Robotic Sy 10.47 (2013).

[18] Vijay, B., and A. Nagabhushana Rao. "Analysis Of Face Recognition-A Case Study On Feature Selection And Feature Normalization." Analysis 3.3 (2013): 971-979.

[19] Ahmed, Nasir, T. Natarajan, and Kamisetty R. Rao. "Discrete cosine transform." Computers, IEEE Transactions on 100.1 (1974): 90-93.

[20] Lee, Byeong. "A new algorithm to compute the discrete cosine transform." Acoustics, Speech and Signal Processing, IEEE Transactions on 32.6 (1984): 12431245. 